\title{
MicroRNA-224 is downregulated in mucinous cystic neoplasms of the pancreas and may regulate tumorigenesis by targeting Jagged1
}

\author{
BEIBEI ZHANG, XIAORONG GUO, JINGXI ZHANG, XIAO LIU, XIANBAO ZHAN and ZHAOSHEN LI \\ Department of Gastroenterology, Changhai Hospital, Second Military Medical University, Shanghai 200433, P.R. China
}

Received November 1, 2013; Accepted June 9, 2014

DOI: $10.3892 / \mathrm{mmr} .2014 .2658$

\begin{abstract}
The underlying malignancy of mucinous cystic neoplasms (MCNs) of the pancreas most commonly results in patients undergoing surgery. The tumorigenesis of MCNs remains elusive and few studies have investigated the role of specific micro (mi)RNAs in MCNs. The present study focused on the expression of miRNA-224 and its putative target gene Jagged1 (Jag1) to examine its role in tumorigenesis and its suitability as a biomarker for MCNs. Paired tissue samples confirmed by surgical pathology were used to screen the miRNAs involved in MCNs with miRNA microarrays $(n=3)$, and to verify the differentially expressed miRNAs $(\mathrm{n}=3)$ and mRNAs of candidate target genes of miRNAs by quantitative polymerase chain reaction ( $\mathrm{qPCR} ; \mathrm{n}=8$ ). Immunohistochemistry was conducted to confirm the expression and location of Jag1 in the neoplastic epithelial cells. Luciferase assays were performed to confirm the direct target gene of miRNA-224. miRNA microarray analysis revealed that two differentially expressed miRNAs were closely associated with tumorigenesis and pancreatic diseases. The qPCR results revealed that miRNA-224 was more significantly aberrantly expressed and the mRNA expression levels of its putative target gene, Jag1, were upregulated. Strong, diffuse cytoplasmic immunohistochemical labeling of Jag1 with occasional nuclear labeling was detected in the mucinous epithelium. Luciferase reporter activity was significantly reduced by co-transfected miRNA-224 mimics and pMIR-Jag1-wild-type, which suggested that miRNA-224 bound to recognition sites in the 3' untranslated region of its target mRNA, Jag1. In conclusion, miRNA-224 was downregulated in MCNs and may regulate tumorigenesis by targeting Jag1. Further studies investigating the role of miRNAs and functional analysis of
\end{abstract}

Correspondence to: Professor Xianbao Zhan or Professor Zhaoshen Li, Department of Gastroenterology, Changhai Hospital, Second Military Medical University, Shanghai 200433, P.R. China E-mail: xianbaozhan@yahoo.com

E-mail: zhsli@81890.net

Key words: mucinous cystic neoplasms of pancreas, microRNA-224, Jagged 1, tumorigenesis epigenetic alterations are required to examine the diagnostic and therapeutic potential of miRNAs in MCNs.

\section{Introduction}

Mucinous cystic neoplasms (MCNs) of the pancreas are rare cystic tumors, $6 \sim 36 \%$ of which may progress to malignancy $(1,2)$. The prevalence of MCN is considerably more frequent in females $(9 \sim 20: 1)(3,4,5)$ and the disease most commonly occurs between 40 and 50 years of age, with a mean age of 47 years (6). Surgical resection may achieve satisfactory outcomes for non-invasive MCNs, while the five-year survival rate of invasive cancer is only 50 60\% (5). Various biomarkers, including carcinoembryonic antigen (CEA), carbohydrate antigen (CA)19-9, CA72-4, and molecular alterations of K-ras mutation obtained by endoscopic ultrasonography-guided fine needle aspirate from pancreatic cysts (7), may be inadequate as predictors of malignancy in MCNs. Patients most commonly undergo resections for evaluating the risk of malignancy.

Micro (mi)RNAs are short, non-coding RNAs that function as potent regulators of gene expression. Epigenetic alterations of miRNAs offer new approaches to the diagnosis and treatment of a variety of human diseases. The emerging roles of miRNAs as oncogenes or tumor suppressors in pancreatic cancer compared with chronic pancreatitis $(8,9)$, pancreatic intraepithelial neoplasms (10), intraductal papillary mucinous neoplasms (11) or normal pancreas (8), including miRNA-21 (12,13), miRNA-34a (13) and miRNA-155 (14). It is therefore valid to suggest that miRNA expression profiles and regulation mechanisms may also be involved in the tumorigenesis in MCNs. However, few epigenetic studies have been conducted investigating the miRNA expression profiles in this type of disease.

The aim of the present study was to screen aberrant gene expression in MCNs using miRNA microarrays, to validate the expression levels of candidate miRNAs using quantitative polymerase chain reaction (qPCR) and to confirm the target genes of miRNAs using bioinfomatics and luciferase assays.

\section{Materials and methods}

Tissues samples. Fresh tissues from pancreatic resections were obtained from eight female patients (25-67 years old; mean, 44 years) who underwent distal pancreatectomy at Changhai hospital between May 1st and December 31st, 2011 (Shanghai, 
Table I. Characteristics of eight patients with mucinous cystic neoplasms confirmed by pathohistological diagnosis.

\begin{tabular}{|c|c|c|c|c|c|c|c|}
\hline & Gender & $\begin{array}{c}\text { Age } \\
\text { (years) }\end{array}$ & $\begin{array}{l}\text { Tumor size } \\
\text { (cm) }\end{array}$ & Location & $\begin{array}{c}\text { CEA } \\
(10 \mathrm{ng} / \mathrm{ml})\end{array}$ & $\begin{array}{l}\text { CA19-9 } \\
(37 \mathrm{U} / \mathrm{ml})\end{array}$ & Pathohistological diagnosis \\
\hline 1 & $\mathrm{~F}$ & 45 & $4 \times 5 \times 3$ & Body and tail & 1.06 & 9.64 & Cystadenoma \\
\hline 2 & $\mathrm{~F}$ & 41 & $4 \times 3 \times 2$ & Tail & 1.56 & 30.03 & Cystadenoma \\
\hline 3 & $\mathrm{~F}$ & 25 & $6 \times 5 \times 3$ & Tail & 1.31 & 31.52 & Cystadenoma \\
\hline 4 & $\mathrm{~F}$ & 30 & $8 \times 8 \times 7$ & Body and tail & 0.54 & 11.22 & Cystadenoma \\
\hline 5 & $\mathrm{~F}$ & 47 & $8 \times 4 \times 3$ & Tail & 1.00 & $<2.00$ & Cystadenoma \\
\hline 6 & $\mathrm{~F}$ & 44 & $11 \times 3 \times 3$ & Body & 2.64 & 14.65 & Cystadenoma \\
\hline 7 & $\mathrm{~F}$ & 67 & $3 \times 2 \times 1$ & Tail & 2.07 & $<2.00$ & Cystadenoma \\
\hline 8 & $\mathrm{~F}$ & 51 & $10 \times 9 \times 4$ & Tail & 2.09 & 62.11 & Cystadenoma borderline \\
\hline
\end{tabular}

CEA, carcinoembryonic antigen; CA19-9, carbohydrate antigen 19-9.

China) and the corresponding adjacent normal tissues were collected for comparison. Written informed consent was obtained from the patients. The clinicopathological characteristics are summarized in Table I. All of the specimens were immediately stored in liquid nitrogen for RNA extraction prior to the confirmation of the histological types by the Department of Pathology (Changhai Hospital, Second Military Medical University, Shanghai, China). The eight MCNs included seven cystadenoma neoplasms and one borderline neoplasm. The use of the tissue samples for all of the experiments was approved by all the patients and by the Ethics Committee of Changhai Hospital (Shanghai, China).

RNA extraction and miRNA microarrays. miRNA isolation from tumors and corresponding adjacent normal tissues was performed with a mirVana ${ }^{\mathrm{TM}}$ RNA Isolation kit (Ambion, Austin, TX, USA), according to the manufacturer's instructions and stored at $-80^{\circ} \mathrm{C}$ prior to analysis. To determine the quality, miRNAs were further assessed using the Agilent 2100 bioanalyzer with the RNA 6000 Nano LabChip reagent set (Agilent Technologies, Santa Clara, CA, USA). Gene expression profiling was performed with Agilent's human miRNA microarrays version $16.0 \mathrm{v}$ (based on Sanger miRBase version 16.0, carrying 1347 human miRNAs and 142 human viral miRNAs; http://microrna.sanger.ac.uk). Each miRNA is represented by 40 probes on the array. A total of $100 \mathrm{ng}$ of total RNA was dephosphorylated, labeled by ligation with one cyanine 3-pCp molecule to the 3' end of the RNA molecule and was hybridized on the miRNA microarray slides at $55^{\circ} \mathrm{C}$ for $20 \mathrm{~h}$. Following microarray washing, the sliders were scanned and detection of the labeled miRNAs on the microarrays was performed according to the instructions from Agilent Technologies. Scanner data files from miRNA microarrays were analyzed by GeneSpring GX9.0 (Agilent Technologies). The hybridization signals that failed to exceed the average background value by $>2$ standard deviations (SDs) were excluded from analysis.

Taqman probe-based qPCR analysis of miRNAs. miRNA expression changes identified by miRNA microarrays were confirmed by Taqman miRNA assays and normalization was performed with the small nuclear RNA U6 (Applied
Table II. Primer of three candidate genes mRNA sequences.

$\begin{array}{lll}\text { Jagged1 } & \text { Sense } & \text { 5-GCGTGGGATTCCAGTAATG-3 } \\ & \text { Antisense } & \text { 5-TTACAGCCAAAGCCATAGTAG-3 } \\ \text { L1CAM } & \text { Sense } & \text { 5-AAATGGCTGTGAAGACCA ATG-3 } \\ & \text { Antisense } & \text { 5-CCTGGGTGTCCTCCTTATC-3 } \\ \text { SMAD4 } & \text { sense } & \text { 5-CCAGGATCAGTAGGTGGAAT-3 } \\ & \text { Antisense } & \text { 5-GTCTAAAGGTTGTGGGTCTG-3 }\end{array}$

L1CAM, cell adhesion molecule L1.

Biosystems, Foster City, CA, USA) according to the manufacturer's instructions. miRNAs were isolated using the method described above. cDNAs were generated using an ABI 9700 PCR instrument (Applied Biosystems). qPCR was performed on a lightcycler 480 type II instrument (Roche Applied Science, Penzberg, Germany). qPCR was performed in triplicate and the relative expression was calculated by the $2^{-\Delta \Delta \mathrm{Ct}}$ method.

Bioinformatics of target identification. For identification of putative target genes of differentially expressed miRNAs, the miRanda engine (predicted microRNA targets and target downregulation scores; http://www.microrna.org/microrna/home. do) and miRGEN database (a database of microRNA targets and target downregulation scores; http:www.diana.pcbi.upenn. edu/miR Gen/v3/miRGen.html) were used. For analysis of Kegg signal pathways and GO terms of candidate genes, the online analytical processing of MAS (CapitalBio Molecule Annotation System; http://bioinfo.capitalbio.com/mas3/) and DAVID system (Database for Annotation, Visualization and Integrated Discovery; http://david.abcc.ncifcrf.gov/) were performed. The Venn diagram (http://bioinfogp.cnb.csic. es/tools/venny/index.html), an interactive tool for comparing lists using Venn Diagrams, was used to integrate data for further functional studies.

SYBR Green-based qPCR analysis of mRNAs. To detect the expression of the candidate mRNAs, reverse-transcription and qPCR amplification of isolated RNA were performed by using a PrimeScript 1st Strand cDNA Synthesis kit and SYBR 
Table III. Differentially expressed microRNAs with microarrays in mucinous cystic neoplasms.

\begin{tabular}{lccc}
\hline Gene Name & Fdr & Fold-change & P-value \\
\hline hsa-miR-197 & 0.99178 & 0.42 & 0.01557 \\
hsa-miR-224 & 0.99178 & 0.37 & 0.02595 \\
\hline
\end{tabular}

Fold change compared with adjacent normal tissues. hsa, Homo sapiens; miR, microRNA; Fdr, false discovery rate.

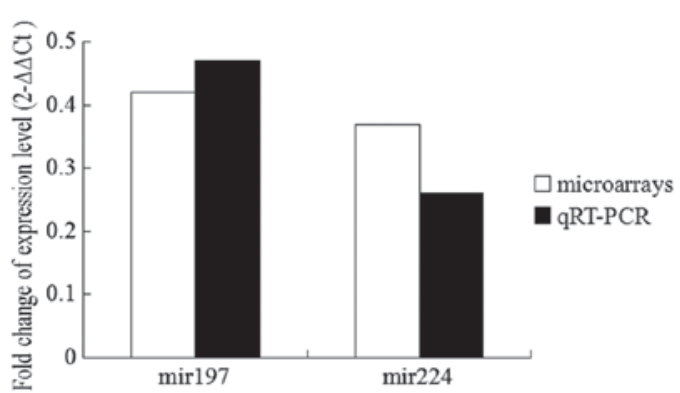

Figure 1. The results of qRT-PCR $(n=8)$ were matched with the microRNA microarrays $(n=3)$. The $y$-axis represents the expression levels $\left(2^{-\Delta \Delta C t}\right)$. The difference was not statistically significant $(\mathrm{P}=0.351$ by paired sample test, vs. adjacent normal tissues.. qRT-PCR, quantitative real-time polymerase chain reaction; $\mathrm{miR}$, microRNA.

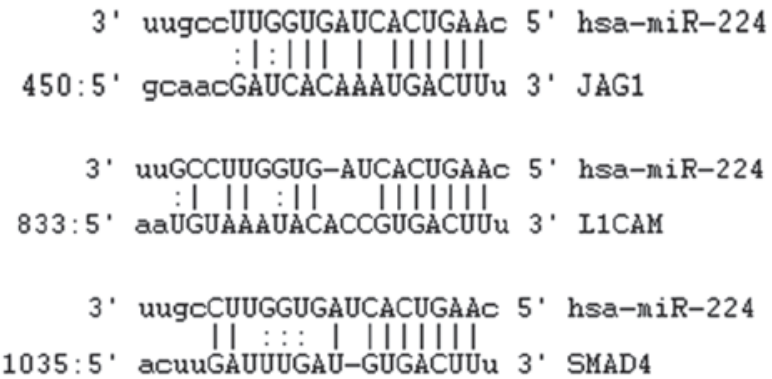

Figure 2. miR-224 binding site in 3'UTR of JAG1, L1CAM and SMAD4 were predicted by miRanda (http://www.microrna.org/microrna/home.do). miR, microRNA; L1CAM, cell adhesion molecule L1; hsa, Homo sapiens.

Premix Ex Taq ${ }^{\mathrm{TM}}$ kit (Takara Bio, Inc., Shiga, Japan) according to the manufacturer's instructions. The specific primer sequence sets of mRNAs (15) for qPCR are summarized in Table II. GAPDH, 5'-ACCACAGTCCATGCCATCAC-3' and 5'-TCCACCACCCTGTTGCTGTA-3' were used to normalize the data. cDNAs were generated using the GeneAmp 9700 PCR System (Applied Biosystems). qPCR was performed on a 7500HT Fast Real-Time PCR System (Applied Biosystems). qPCR was performed in triplicate, including no template controls. The relative expression was calculated by the $2^{-\Delta \Delta \mathrm{Ct}}$ method and the data were analyzed with Real-Time StatMiner 7500 v.2.0.6 (Integromics, Granada, Spain).

Immunohistochemistry. The hematoxylin and eosin-stained slides from each of the cases were screened by light microscopy (OLYMPUS BX51; Olympus, Tokyo, Japan) for sections containing an MCN and adjacent normal pancreas tissue. Unstained $4-\mu \mathrm{m}$ sections were then cut from the paraffin block selected for each case and deparaffinized by routine techniques. Next, the slides were treated with $1 \mathrm{X}$ sodium citrate buffer prior to steaming for $12 \mathrm{~min}$ at $80^{\circ} \mathrm{C}$. The slides were then cooled to incubate with a 1:50 dilution of polyclonal antibody to Jag1 protein (H-66 sc-11376; dilution, 1:100; Santa Cruz Biotechnology, Inc., Santa Cruz, CA, USA) and detected by diaminobenzidine staining.

Luciferase reporter assays. The full-length 3' UTR of Jag1-wild-type (WT) was amplifed from OVCR cells using the primers listed: Jag1 3'UTR-WT, sense 5'-GACTAGTTTGCTGACTTAGAATCCCTGTGTT-3' and antisense 5'-CCCAAGCTTGCCATACGCTTACAATG C TA T CA A-3'; mutant ( Mut $) 1, \quad$ s ense 5'-GACTAGTTTGCTGTCACAGAATCCCTGTGTT-3' and antisense identical to WT; MUT2, sense 5'-GACTAGTCGATCACAAACGTCTGTATTATTTA-3' and antisense identical to WT. The nucleotides in bold indicate the conserved sequence of Jag1. The PCR products were gel-purified using the Zymoclean ${ }^{\mathrm{TM}}$ Gel DNA Recovery kit (Zymo Research, Irvine, CA, USA), cloned into pMD-18T vectors (Takara Bio, Inc.) and sequenced to ensure fidelity. Separate plasmids containing the Jag1 3'UTR with mutated seed regions for the predicted miRNA-224 binding sites were also constructed as negative controls. Following sequence verification, the WT or Mut Jag1 3'UTRs were ligated between the SpeI and HindIII restriction sites (SpeI GACTAGT; HindIII CCCAAGCTT) of the reporter plasmid pMIR-Report (Promega Corporation, Madison, WI, USA), which contained correctly oriented inserts (pMIR-Jag1-WT and pMIR-Jag1-Mut). A total of $1 \times 10^{5}$ cells/well were seeded onto 48-well plates on the day prior to transfection, then transfected with miRNA mimics or negative control as described above. The constructed pMIR-reporter vectors and internal control pRL-TK vectors were co-transfected into human embryonic kidney 293T cells by using Lipofectamine 2000 (Invitrogen Life Technologies, Carlsbad, CA, USA) together with miRNA mimics or negative transfection (cells not transfected with miRNA mimics). The 293T cell line was donated by Professor Shanrong Liu (Changhai Hospital, Second Military Medical University, Shanghai, China). The cells were grown in DMEM (Invitrogen Life Technologies) supplemented with $10 \%$ fetal bovine serum (Invitrogen Life Technologies) and $1 \%$ antibiotics $(100 \mathrm{U} / \mathrm{ml}$ penicillin and $100 \mu \mathrm{g} / \mathrm{ml}$ streptomycin; Invitrogen Life Technologies), which were cultured at $37^{\circ} \mathrm{C}$ in a $5 \% \mathrm{CO}_{2}$ atmosphere. The cell line was selected according to the study by Yao et al (16) and is commonly used for transfection. The luciferase reporter assay was performed using a Dual-Glo luciferase assay kit (Promega Corporation) following the manufacturer's instructions.

Statistical analysis. Statistical analyses were performed using SPSS 16.0 software (SPSS, Inc., Chicago, IL, USA). Limma was used to analyze miRNA microarray data since it was suitable for small samples. The differences between the experimental and control groups were analyzed by paired samples test or non-parametric test (Wilcoxon test) as appropriate. Luciferase reporter assay data are expressed as the 
A

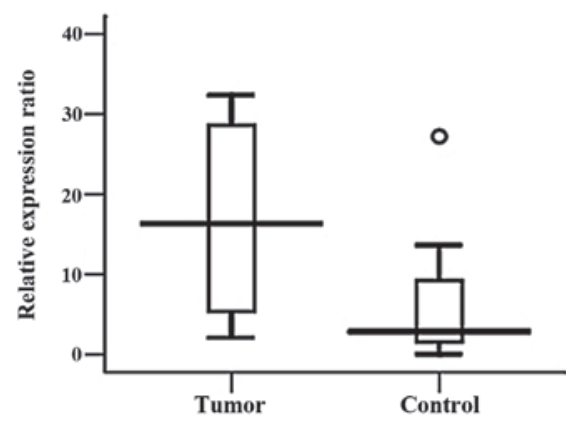

B

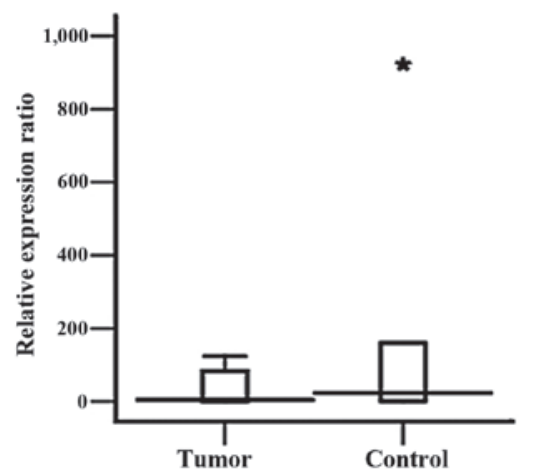

C

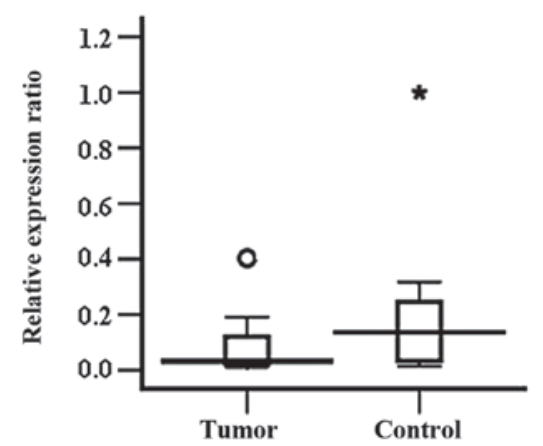

Figure 3. mRNA expression levels of paired samples of (A) Jag1 ( $\mathrm{P}=0.093)$, (B) L1CAM ( $\mathrm{P}=0.484)$ and (C) SMAD4 $(\mathrm{P}=0.03)$ are demonstrated (non-parametric test, $\mathrm{n}=8$ ). Jag1 was upregulated and the median relative expression levels of the neoplasms (16.337) were notably higher than those of the normal controls (2.843), although P $>0.05$. L1CAM and SMAD4 were downregulated, contrary to the hypothesis of this study. Jag1, Jagged1; L1CAM, cell adhesion molecule L1.

mean \pm standard deviation of quadruplicate determinations. $\mathrm{P}<0.05$ was considered to indicate a statistically significant difference.

\section{Results}

Identification of miRNAs differentially expressed in MCNs. A total of three paired samples of MCNs with their corresponding adjacent normal tissues (NT) were qualified for miRNA microarray analysis. Two samples were cystadenoma and the remaining was borderline. miRNA-197 and miRNA-224 were at least two-fold downregulated in MCNs vs. NT as indicated by miRNA microarrays (Limma test; Table III). Next, the qPCR results confirmed the microarray data (paired sample test, $\mathrm{P}=0.351 ;$ Fig. 1). Following the assessment of two miRNAs, miRNA-224 was selected to explore its function, as it was more significantly downregulated and it was reported that miRNA-224 targeted Smad4 (16), which had previously
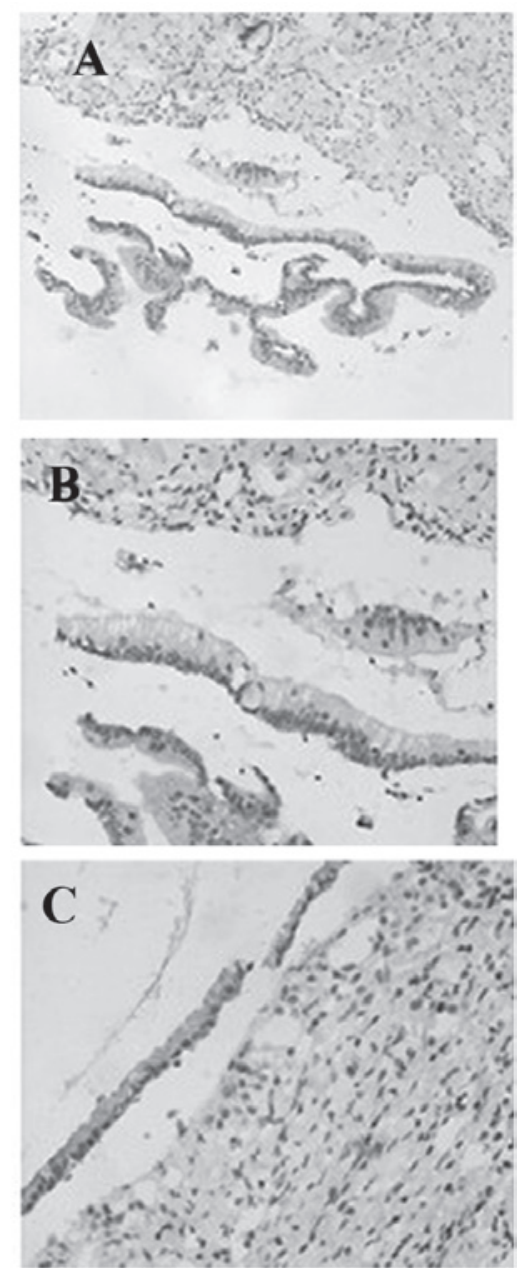

Figure 4. Two different mucinous cystic neoplasms with strong and uniform cytoplasmic and nuclear Jag1 labeling in the neoplastic epithelium compared with their underlying ovarian-like stroma. (A) Images at a magnification of x200 and (B) x400 were from the same sample of a mucinous cystadenoma. (C) Images representing another borderline mucinous cystadenoma (x400).

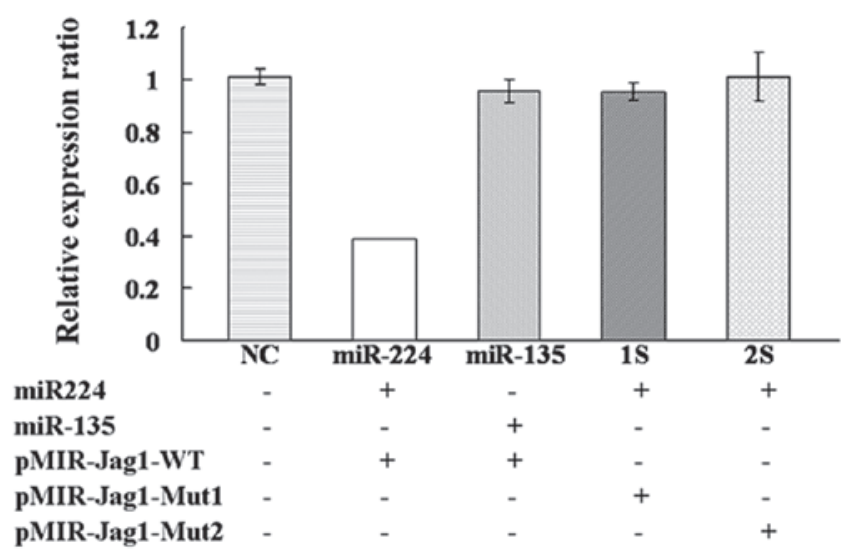

Figure 5. Luciferase reporter assays. At $24 \mathrm{~h}$ following transfection with 40 pmol of mimics miR-224 or miR-135, a reporter plasmid containing Jag1 (WT-3'UTR, Mut1-3'UTR or Mut2-3'UTR), and a plasmid expressing Renilla luciferase (pRL-TK) were co-transfected into 293T cells. Firefly luciferase activity was normalized to Renilla luciferase. Three independent experiments were performed, and representative data are demonstrated (independent-samples t-test; all of the data are expressed as the mean \pm standard deviation; $\mathrm{P}=0.005, \mathrm{NC}$ vs. $\mathrm{miR} 224 ; \mathrm{P}=0.011$, mir224 vs. mir135; $\mathrm{P}=0.007$, mir224 vs. Mut1; $\mathrm{P}=0.045, \operatorname{miR} 224$ vs. Mut2; $\mathrm{P}<0.05$ was considered to indicate a statistically significant difference). miR, microRNA; NC, negative control; Mut, mutant; WT, wild-type; UTR, untranslated region. 
been correlated with MCNs (17) and shown to be involved in invasive adenocarcinoma of the pancreas (18).

miRNA-224 may be associated with tumorigenesis in MCNs by regulating Jag1. The present study focused on potential mRNA targets of miRNA-224 and selected Jag1, L1CAM and SMAD4 (putative binding site in miRanda; Fig. 2) as the candidate genes by bioinformatics, according to the results that their mRNA was overexpressed in MCNs according to a study by Fukushima et al (15). These putative target mRNAs were also found distributed in the Notch/Wnt (19), nuclear factor- $\kappa \mathrm{B}$ (20) and transforming growth factor (21) signaling pathways, which were correlated with pancreatic diseases (22), tumorigenesis and tumor progression (23-25). The present study then focused more on Jag1 expression, as its mRNA levels were upregulated in tumor tissues as compared with normal tissue samples according to $\mathrm{qPCR}$ analysis (non-parametric test, $\mathrm{P}=0.093$; Fig. 3A), while L1CAM ( $\mathrm{P}=0.484$; Fig. 3B) and SMAD4 ( $\mathrm{P}=0.03$; Fig. $3 \mathrm{C})$ were downregulated. Furthermore, it was identified that Jag1 protein expression was markedly upregulated in the cytoplasm and nucleus of neoplastic epithelium (Fig. 4). No detectable stroma labeling was observed according to the images. This suggested that Jag1 may have an important role in the tumorigenesis of MCNs. It was hypothesized that the upregulation of Jag1 may be due to the post-transcriptional regulation of miRNA-224 according to their negative correlation and immunohistochemical staining

miRNA-224 target Jag1 mRNA 3'UTR confirmed by luciferase reporter assays. To confirm the hypothesis of miRNA-224 targeting the 3'UTR of Jag1 and therefore regulating Jag1 expression, the miRNA-224 binding sequence present at the 3'UTR of Jag1 mRNA (WT-3'UTR) or its Mut (Mut-3'UTR) was cloned downstream of the firely luciferase reporter gene and co-transfected with miRNA-224 mimics or miRNA-135 mimics into $293 \mathrm{~T}$ cells. Relative luciferase activity was significantly reduced by miRNA-224 mimics, but not by miRNA-135 (Fig. 5). Furthermore, when the miRNA-224 mimics were co-transfected, the relative luciferase activity of a reporter containing WT-3'UTR was significantly suppressed by $38 \%(\mathrm{P}<0.005)$ compared with that of a reporter containing Mut-3'UTR, which was unaffected by simultaneous transfections with the negative control. This result was reproducible in the additional two independent experiments, suggesting that miRNA-224 targets the miRNA-224 binding sequence at the 3'UTR of Jag1.

\section{Discussion}

It has been reported that miRNA-224 is upregulated in a variety of tumor types $(18,26)$ and effects cell invasion, migration and proliferation, resulting in the dysregulation of cellular processes that ultimately lead to tumorigenesis. However, miRNA-224 was downregulated in the present study and may have a role as a dynamic regulator (27) in MCNs. Whether its dysregulation is consistent with the function as an oncogene or tumor-suppressor remains elusive. Next, Jag1 was selected as a candidate gene by focusing on bioinformatics and the mRNA microarray results of MCNs (15).
The qPCR results analyzing the mRNA expression levels of Jag1 were in accordance with the mRNA microarrays, which indicated a negative correlation between miRNA-224 and Jag1, and the immunohistochemical staining also indicated that Jag1 expression may have a role in MCNs. To validate the function of these miRNAs, the mimics or pre-miRNAs were transfected into an MCN cell line and the resulting changes in the mRNA and protein expression of Jag1 were then detected. However, with MCN cell lines being unavailable, it was not possible to observe the epigenomic changes of overexpression or inhibition of miRNA-224 or Jag1 directly. The attempt to establish the MCC1 cell line using the method described previously by Sorio et al (28) was unsuccessful, and therefore, further studies are required to examine the validity of these results using this technique.

Jag1 has a genomic location in chromosome 20p12.1-p11.23 and is involved in the notch/Wnt signal pathway (29). The present study demonstrated that there was a correlation between Jag1 protein expression and neoplastic epithelium, although the direct evidence was not obtained from an MCN cell line. Prior to experimentation, several studies were retrospectively reviewed, including those investigating miRNAs targeting the Jag1 3'UTR, such as miRNA-21, as an oncomir, which decreased Jag1 expression levels in MCF-7 breast cancer cells (30); miRNA-34a, as a known tumor suppressor gene, inhibited abnormal cell growth through regulation of the Notch pathway in cervical carcinoma and choriocarcinoma cells (31); miRNA-200c and miRNA-141 directly inhibited Jag1, impeding the proliferation of human metastatic prostate cancer cells (32) and coordinated the upregulation of Jag1 and zinc finger E-box binding homolog 1, associated with reduced miRNA-200 expression in the aggressive pancreatic adenocarcinoma and basal type of breast cancer (33). The correlation between these miRNAs and Jag1 demonstrated that miRNAs operate in a highly complex regulatory network. Next, it was identified that the function of the Jag1 protein in tumorigenesis was contradictory to other studies. Ban et al (34) demonstrated that the activation of the Notch signaling pathway had tumor suppressive-like effects, leading to nuclear accumulation of p53 activation and cell cycle arrest in Ewing's sarcoma family of tumors. Sasaki et al (35) identified a specific binding site for the p63 protein, which was a suppressor gene p53 family member, in the second intron of the Jag1 gene and demonstrated that p63 may activate Notch signaling. The high expression levels of Jag1 mRNA and protein expression were indicative of a poor patient outcome in breast cancer (36). Notably, Jag1 acts as an inhibitor of Notch signaling during embryonic pancreas development, but an activator of Notch signaling postnatally (37). Focusing on pancreatic disease, it was noted that Jag1 was implicated in epithelial-mesenchymal transition (38), ductal malformation and pancreatitis (39). In MCNs, Jag1 was upregulated by 4.8 -fold in the neoplasmic tissues vs. the non-neoplasmic tissues (40), and therefore, the present study focused on Jag1 and examined its potential correlation with miRNA-224 by a luciferase reporter assay. To examine this hypothesis, miRNA-224/miRNA-135 mimics and pMIR-Jag1-WT/Mut1/Mut2 were co-transfected into 293T cells, and the luciferase activity of the reporter containing WT-3'UTR was significantly suppressed compared with the 
samples transfected with the other vectors. However, the function of endogenic miRNA-224 was not verified in MCNs as is mentioned above.

However, MCNs are relatively uncommon neoplasms compared with pancreatic cancer and the small number of tumors examined, to some extent, affected the data. Besides, without laser capture microdissection (41), tissue-specific miRNAs should be distinguished between the neoplastic epithelial cells originating in the duct and stroma cells. Selecting the control sample was another problem that could not be neglected, as normal tissues of the pancreas not belonging to the same person were selected in several studies. Neoplasms vs. adjacent normal tissues $(42,43)$, compared with neoplasms vs. normal pancreata (8), appear to be more reasonable, since it eliminates the individual differences in the present study.

In conclusion, it was firstly demonstrated that miRNA-224 was downregulated in MCNs using microarrays and qPCR, and Jag1, which were confirmed that expressed in the neoplastic tissues, was one of its target genes, as determined by luciferase assay results. The results also indicated that miRNAs may be implicated in tumorigenesis and the tumor progression of MCNs. However, further studies are required to validate the function of both miRNA-224 and Jag1 in MCNs in established MCN cell lines.

\section{Acknowledgements}

The study was supported by Innovation Program of Shanghai Municipal Education Commission (11ZZ72).

\section{References}

1. Crippa S, Fernández-Del CC, Salvia R, et al: Mucin-producing neoplasms of the pancreas: an analysis of distinguishing clinical and epidemiologic characteristics. Clin Gastroenterol Hepatol 8: 213-219, 2010.

2. Testini M, Gurrado A, Lissidini G, Venezia P, Greco L and Piccinni G: Management of mucinous cystic neoplasms of the pancreas. World J Gastroenterol 16: 5682-5692, 2010.

3. Grogan JR, Saeian K, Taylor AJ, Quiroz F, Demeure MJ and Komorowski RA: Making sense of mucin-producing pancreatic tumors. AJR Am J Roentgenol 176: 921-929, 2001.

4. Crippa S, Salvia R, Warshaw AL, et al: Mucinous cystic neoplasm of the pancreas is not an aggressive entity: lessons from 163 resected patients. Ann Surg 247: 571-579, 2008.

5. Matthaei H, Schulick RD, Hruban RH and Maitra A: Cystic precursors to invasive pancreatic cancer. Nat Rev Gastroenterol Hepatol 8: 141-50, 2011.

6. Goh BK, Tan YM, Chung YF, et al: A review of mucinous cystic neoplasms of the pancreas defined by ovarian-type stroma: clinicopathological features of 344 patients. World J Surg 30 2236-2245, 2006.

7. Sawhney MS, Devarajan S, O'Farrel P, et al: Comparison of carcinoembryonic antigen and molecular analysis in pancreatic cyst fluid. Gastrointest Endosc 69: 1106-1110, 2009.

8. Bloomston M, Frankel WL, Petrocca F, et al: MicroRNA expression patterns to differentiate pancreatic adenocarcinoma from normal pancreas and chronic pancreatitis. JAMA 297: 1901-1908, 2007.

9. Szafranska AE, Doleshal M, Edmunds HS, et al: Analysis of microRNAs in pancreatic fine-needle aspirates can classify benign and malignant tissues. Clin Chem 54: 1716-1724, 2008.

10. Yu J, Li A, Hong SM, Hruban RH and Goggins M: MicroRNA alterations of pancreatic intraepithelial neoplasias. Clin Cancer Res 18: 981-992, 2012.

11. Remmers N, Bailey JM, Mohr AM and Hollingsworth MA: Molecular pathology of early pancreatic cancer. Cancer Biomark 9: 421-440, 2011.
12. du Rieu MC, Torrisani J, Selves J, et al: MicroRNA-21 is induced early in pancreatic ductal adenocarcinoma precursor lesions. Clin Chem 56: 603-612, 2010.

13. Jamieson NB, Morran DC, Morton JP, et al: MicroRNA molecular profiles associated with diagnosis, clinicopathologic criteria, and overall survival in patients with resectable pancreatic ductal adenocarcinoma. Clin Cancer Res 18: 534-545, 2012.

14. Habbe N, Koorstra JB, Mendell JT, et al: MicroRNA miR-155 is a biomarker of early pancreatic neoplasia. Cancer Biol Ther 8: 340-346, 2009.

15. Fukushima N, Sato N, Prasad N, Leach SD, Hruban RH and Goggins M: Characterization of gene expression in mucinous cystic neoplasms of the pancreas using oligonucleotide microarrays. Oncogene 23: 9042-9051, 2004.

16. Yao G, Yin M, Lian J, et al: MicroRNA-224 is involved in transforming growth factor-beta-mediated mouse granulosa cell proliferation and granulosa cell function by targeting Smad4. Mol Endocrinol 24: 540-551, 2010

17. Izeradjene K, Combs C, Best M, et al: $\mathrm{Kras}(\mathrm{G} 12 \mathrm{D})$ and Smad4/Dpc4 haploinsufficiency cooperate to induce mucinous cystic neoplasms and invasive adenocarcinoma of the pancreas. Cancer Cell 11: 229-243, 2007.

18. Mees ST, Mardin WA, Sielker S, et al: Involvement of CD40 targeting miR-224 and miR-486 on the progression of pancreatic ductal adenocarcinomas. Ann Surg Oncol 16: 2339-2250, 2009.

19. Rodilla V, Villanueva A, Obrador-Hevia A, et al: Jag1 is the pathological link between Wnt and Notch pathways in colorectal cancer. Proc Natl Acad Sci USA 106: 6315-6320, 2009.

20. Kiefel H, Bondong S, Erbe-Hoffmann N, et al: L1CAM-integrin interaction induces constitutive NF-kappaB activation in pancreatic adenocarcinoma cells by enhancing IL-1beta expression. Oncogene 29: 4766-4778, 2010.

21. Levy L and Hill CS: Smad4 dependency defines two classes of transforming growth factor $\{$ beta\} (TGF- $\{$ beta $\}$ ) target genes and distinguishes TGF-\{beta\}-induced epithelial-mesenchymal transition from its antiproliferative and migratory responses. Mol Cell Biol 25: 8108-8125, 2005.

22. Maniati E, Bossard M, Cook N, et al: Crosstalk between the canonical NF- $\kappa B$ and Notch signaling pathways inhibits Ppar $\gamma$ expression and promotes pancreatic cancer progression in mice. J Clin Invest 121: 4685-4699, 2011.

23. Wang Z, Banerjee S, Ahmad A, et al: Activated K-ras and INK4a/Arf deficiency cooperate during the development of pancreatic cancer by activation of Notch and NF- $\mathrm{KB}$ signaling pathways. PLoS One 6: e20537, 2011.

24. Bao B, Wang Z, Ali S, et al: Notch-1 induces epithelial-mesenchymal transition consistent with cancer stem cell phenotype in pancreatic cancer cells. Cancer Lett 307: 26-36, 2011.

25. Vaccaro V, Melisi D, Bria E, et al: Emerging pathways and future targets for the molecular therapy of pancreatic cancer. Expert Opin Ther Targets 15: 1183-1196, 2011.

26. Prueitt RL, Yi M, Hudson RS, et al: Expression of microRNAs and protein-coding genes associated with perineural invasion in prostate cancer. Prostate 68: 1152-1164, 2008.

27. Davalos V, Moutinho C, Villanueva A, et al: Dynamic epigenetic regulation of the microRNA-200 family mediates epithelial and mesenchymal transitions in human tumorigenesis. Oncogene 31: 2062-2074, 2012.

28. Sorio C, Capelli P, Lissandrini D, et al: Mucinous cystic carcinoma of the pancreas: a unique cell line and xenograft model of a preinvasive lesion. Virchows Arch 446: 239-245, 2005.

29. Katoh M and Katoh M: Notch ligand, JAG1, is evolutionarily conserved target of canonical WNT signaling pathway in progenitor cells. Int J Mol Med 17: 681-685, 2006.

30. Selcuklu SD, Donoghue MT, Kerin MJ and Spillane C: Regulatory interplay between miR-21, JAG1 and 17beta-estradiol (E2) in breast cancer cells. Biochem Biophys Res Commun 423: 234-239, 2012.

31. Pang RT, Leung CO, Ye TM, et al: MicroRNA-34a suppresses invasion through downregulation of Notch1 and Jag1 in cervical carcinoma and choriocarcinoma cells. Carcinogenesis 31: 1037-1044, 2010.

32. Steg AD, Katre AA, Goodman B, et al: Targeting the notch ligand JAGGED1 in both tumor cells and stroma in ovarian cancer. Clin Cancer Res 17: 5674-5685, 2011.

33. Brabletz S, Bajdak K, Meidhof S, et al: The ZEB1/miR-200 feedback loop controls Notch signalling in cancer cells. EMBO J 30: 770-782, 2011. 
34. Ban J, Bennani-Baiti IM, Kauer M, et al: EWS-FLI1 suppresses NOTCH-activated p53 in Ewing's sarcoma. Cancer Res 68: 7100-7109, 2008.

35. Sasaki Y, Ishida S, Morimoto I, et al: The p53 family member genes are involved in the Notch signal pathway. J Biol Chem 277: 719-724, 2002.

36. Dickson BC, Mulligan AM, Zhang H, et al: High-level JAG1 mRNA and protein predict poor outcome in breast cancer. Mod Pathol 20: 685-693, 2007.

37. Golson ML, Le Lay J, Gao N, et al: Jag1 is a competitive inhibitor of Notch signaling in the embryonic pancreas. Mech Dev 126: 687-699, 2009.

38. Bao B, Wang Z, Ali S, et al: Notch-1 induces epithelial-mesenchymal transition consistent with cancer stem cell phenotype in pancreatic cancer cells. Cancer Lett 307: 26-36, 2011.
39. Golson ML, Loomes KM, Oakey R and Kaestner KH: Ductal malformation and pancreatitis in mice caused by conditional Jag1 deletion. Gastroenterology 136: 1761-1771, 2009.

40. Fukushima N, Sato N, Prasad N, Leach SD, Hruban RH and Goggins M: Characterization of gene expression in mucinous cystic neoplasms of the pancreas using oligonucleotide microarrays. Oncogene 23: 9042-9051, 2004.

41. Tan MH,Nowak NJ,Loor R,etal: Characterization of a new primary human pancreatic tumor line. Cancer Invest 4: 15-23, 1986.

42. Volinia S, Calin GA, Liu CG, et al. A microRNA expression signature of human solid tumors defines cancer gene targets. Proc Natl Acad sci USA 103: 2257-2261, 2006.

43. Zhang S, Hao J, Xie F, Hu X, et al: Downregulation of miR-132 by promoter methylation contributes to pancreatic cancer development. Carcinogenesis 32: 1183-1189, 2011. 